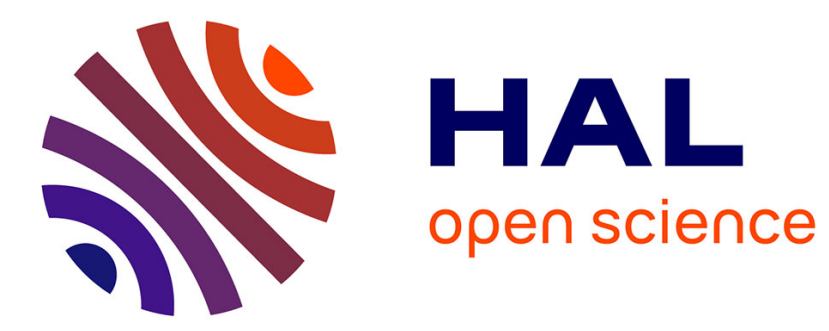

\title{
Detection and localization of a defect in a reverberant plate using acoustic field correlation
}

Lynda Chehami, Emmanuel Moulin, Julien de Rosny, Claire Prada, Olivier Bou Matar, Farouk Benmeddour, Jamal Assaad

\section{- To cite this version:}

Lynda Chehami, Emmanuel Moulin, Julien de Rosny, Claire Prada, Olivier Bou Matar, et al.. Detection and localization of a defect in a reverberant plate using acoustic field correlation. Journal of Applied Physics, 2014, 115, pp.104901-1-7. 10.1063/1.4867522 . hal-00964507

\section{HAL Id: hal-00964507 https://hal.science/hal-00964507}

Submitted on 7 Oct 2021

HAL is a multi-disciplinary open access archive for the deposit and dissemination of scientific research documents, whether they are published or not. The documents may come from teaching and research institutions in France or abroad, or from public or private research centers.
L'archive ouverte pluridisciplinaire HAL, est destinée au dépôt et à la diffusion de documents scientifiques de niveau recherche, publiés ou non, émanant des établissements d'enseignement et de recherche français ou étrangers, des laboratoires publics ou privés. 


\title{
Detection and localization of a defect in a reverberant plate using acoustic field correlation
}

\author{
Lynda Chehami, ${ }^{1}$ Emmanuel Moulin, ${ }^{1, a)}$ Julien de Rosny, ${ }^{2}$ Claire Prada, ${ }^{2}$ \\ Olivier Bou Matar, ${ }^{1}$ Farouk Benmeddour, ${ }^{1}$ and Jamal Assaad ${ }^{1}$ \\ ${ }^{1}$ IEMN UMR CNRS 8520, Université de Valenciennes et du Hainaut-Cambrésis, \\ F-59313 Valenciennes Cedex 9, France \\ ${ }^{2}$ Institut Langevin, ESPCI-CNRS, UMR 7587, 1 rue Jussieu, F-75231 Paris Cedex 05, France
}

(Received 20 December 2013; accepted 22 February 2014; published online 10 March 2014)

\begin{abstract}
Green's function can be retrieved from cross-correlation of a diffuse field generated by noise sources. Today, this important result is the fundamental of several passive imaging techniques. The aim of this paper is to establish the suitability of these methods to detect and locate a defect in a reverberant elastic plate. The relations between the noise cross-correlation function over a few number of noise sources and the imaginary part of the Green's function are derived and numerically validated. Then we show through numerical experiments that this technique is exploitable for defect detection and localization in a differential mode, despite a non-perfect estimation of the Green's functions. Finally, a filtering technique based on the singular value decomposition is shown to improve the detection. (C) 2014 AIP Publishing LLC.
\end{abstract}

[http://dx.doi.org/10.1063/1.4867522]

\section{INTRODUCTION}

Structural health monitoring (SHM) is an ensemble of emerging technologies aiming at helping optimisation in maintenance, reliability, and structures safety, particularly in the aeronautical field. Commonly studied SHM systems involve guided elastic waves, namely Lamb waves, which can propagate over long distances in plates and shells. ${ }^{1,2}$ Unlike standard ultrasound methods (conventional methods or pitch-catch) ${ }^{3}$ used in the active configuration (active sensors emit waves to interrogate the defect present in the structure), a passive SHM system based on ambient noise correlation would not require power-consuming ultrasound emitters. In an aeronautical application, for instance, useful noise could be engine noise or aerodynamic noise.

Several theoretical and experimental studies have demonstrated the possibility of retrieving a relevant information about a propagation medium by using ambient noise. Indeed, when it is diffused or more exactly equipartioned, the Green's functions between two points of a structure can be reconstructed by the correlation of noise field recorded in these two points. Such methods have been studied in several areas such as civil engineering, ${ }^{4}$ seismology,${ }^{5,6}$ medical imaging, ${ }^{7}$ underwater acoustics, ${ }^{8,9}$ volcanology, ${ }^{10}$ ultrasound, ${ }^{11-13}$ helioseismology, ${ }^{14}$ and nondestructive testing. ${ }^{15-17}$

The complexity of this application lies on the nondiffusivity of the ambient field, which leads to an unperfect convergence of correlations towards the Green's functions. As a consequence, localizing, detecting, and imaging defect is a real challenge.

In this paper, we propose an application of the Green's function extraction to passively detect a defect in a thin plate. To this end, we estimate the cross-correlation matrix of the flexural waves generated by random sources and measured

\footnotetext{
a)emmanuel.moulin@univ-valenciennes.fr
}

on a few sensors. The difference between the matrix obtained with and without defect (differential mode) allows to detect and localize the defect. From numerical experiments, we show that despite the imperfect reconstruction of the Green's functions, the detection and localization of a defect might be achieved with only 8 receivers. Thereafter, to improve the sensitivity of detection, an emerging technique of physical acoustic based on time reversal associated to singular value decomposition (DORT method, French acronym for Decomposition of the Time Reversal Operator) ${ }^{18-20}$ is applied to the correlation matrices of the received signals.

This paper is organized as follows: in Sec. II, we show how the Green's functions can be extracted from the averaged correlation matrices when the number of noise sources is limited. Similarly to the works by Weaver and Lobkis for bulk acoustic waves, ${ }^{11,21}$ the derivation is based on a modal expansion of the flexural waves. A quantitative comparison between the averaged correlations and the Green's functions obtained from numerical simulation is thus obtained. Then in Sec. III, a back-propagation algorithm is successfully applied to the correlation matrices for localizing and imaging the defect. Finally, a filtering technique based on the DORT method is applied to reduce noise and to improve the quality of the obtained images.

\section{GREEN'S FUNCTION RETRIEVAL FROM A FINITE NUMBER OF NOISE SOURCES}

\section{A. Theoretical relation between correlation and Green's function}

The normal displacement field $w$ of flexural waves obeys the Kirchhoff-Love equation, ${ }^{22}$

$$
D \Delta^{2} w(\mathbf{r}, t)+\rho_{s}\left[\partial_{t}^{2} w(\mathbf{r}, t)+\frac{1}{\tau_{a}} \partial_{t} w(\mathbf{r}, t)\right]=-s(\mathbf{r}, t),
$$


where $\mathbf{r}$ is the position vector, $D=h^{3} E / 12\left(1-\nu^{2}\right)$ is the bending stiffness, $h$ the plate thickness, $\rho_{s}=\rho h$ the surfacic mass, $\rho$ the volume density, $E$ the Young's modulus, $\nu$ the Poisson's ratio, $\tau_{a}$ the damping time, and $s$ the normal load. The Green's function is solution of the previous wave equation with a Dirac source term

$$
\begin{aligned}
& D \Delta^{2} G\left(\mathbf{r}_{k}^{S}, \mathbf{r}_{l}^{R}, t\right)+\rho_{s}\left[\partial_{t}^{2} G\left(\mathbf{r}_{k}^{S}, \mathbf{r}_{l}^{R}, t\right)+\frac{1}{\tau_{a}} \partial_{t} G\left(\mathbf{r}_{k}^{S}, \mathbf{r}_{l}^{R}, t\right)\right] \\
& \quad=-\delta\left(\mathbf{r}_{k}^{S}-\mathbf{r}_{l}^{R}\right) \delta(t),
\end{aligned}
$$

with $\mathbf{r}_{k}^{S}$ and $\mathbf{r}_{l}^{R}$ the $k$ th source position and the $l$ th receiver position, respectively.

Now, we assume a set of $N_{s}$ decorrelated noise sources with identical power spectrum $\left\langle|q(\omega)|^{2}\right\rangle$. The Fourier transform of the cross-correlation at frequency $\omega$ between field at positions $\mathbf{r}_{l}^{R}$ and $\mathbf{r}_{l^{\prime}}^{R}$ can be expressed in terms of monochromatic Green's functions,

$$
C_{l l^{\prime}}(\omega)=\sum_{k=1}^{N_{S}} G\left(\mathbf{r}_{k}^{S}, \mathbf{r}_{l}^{R}, \omega\right) G^{*}\left(\mathbf{r}_{k}^{S}, \mathbf{r}_{l^{\prime}}^{R}, \omega\right)\left\langle|q(\omega)|^{2}\right\rangle .
$$

The function $C_{l l^{\prime}}(\omega)$ is also called the cross-spectral density matrix. In highly reverberating media, the plate vibration shows strong resonating modes. The mode at angular frequency $\omega_{n}$ is denoted $\phi_{n}(\mathbf{r})$. We can show that Green's functions can be decomposed over these eigen-modes. ${ }^{23}$ In the frequency domain, this decomposition yields

$$
G\left(\mathbf{r}_{k}^{S}, \mathbf{r}_{l}^{R}, \omega\right)=\frac{1}{\rho_{s}} \sum_{n} \frac{\phi_{n}\left(\mathbf{r}_{k}^{S}\right) \phi_{n}\left(\mathbf{r}_{l}^{R}\right)}{\left(\omega^{2}-\omega_{n}^{2}\right)-j \frac{\omega}{\tau_{a}}} .
$$

By injecting Eq. (4) into Eq. (3), and remarking that the imaginary part of $G\left(\mathbf{r}_{k}^{S}, \mathbf{r}_{l}^{R}, \omega\right)$ is given by

$$
\Im G\left(\mathbf{r}_{k}^{S}, \mathbf{r}_{l}^{R}, \omega\right)=\frac{\omega}{\rho_{s} \tau_{a}} \sum_{n} \frac{\phi_{n}\left(\mathbf{r}_{k}^{S}\right) \phi_{n}\left(\mathbf{r}_{l}^{R}\right)}{\left(\omega^{2}-\omega_{n}^{2}\right)^{2}+\frac{\omega^{2}}{\tau_{a}^{2}}},
$$

the cross-spectral density matrix can be expressed as follows:

$$
C_{l l^{\prime}}(\omega)=2 j \Im G\left(\mathbf{r}_{l}^{R}, \mathbf{r}_{l^{\prime}}^{R}, \omega\right) F(\omega)+N(\omega),
$$

with

$$
F(\omega)=\frac{\left\langle|q(\omega)|^{2}\right\rangle N_{S} \overline{\phi^{2}} \tau_{a}}{2 j \omega \rho_{s}},
$$

where $\overline{\phi^{2}}$ is the spatial mathematical expectation of $\left|\phi_{n}(\mathbf{r})\right|^{2}$, which is close to the inverse of the plate area $S$ because the modes are normalized, i.e., $\int\left|\phi_{n}(\mathbf{r})\right|^{2} d^{2} \mathbf{r}=1$. The expression of $N(\omega)$ then corresponds to the remaining terms that are not included in $2 j \Im G\left(\mathbf{r}_{l}^{R}, \mathbf{r}_{l^{\prime}}^{R}, \omega\right) F(\omega)$

$$
\begin{aligned}
N(\omega)= & \left(\sum_{n \neq n^{\prime}, k} \frac{\phi_{n}\left(\mathbf{r}_{k}^{S}\right) \phi_{n}\left(\mathbf{r}_{l}^{R}\right) \phi_{n^{\prime}}\left(\mathbf{r}_{k}^{S}\right) \phi_{n^{\prime}}\left(\mathbf{r}_{l^{\prime}}^{R}\right)}{\left.\left[\omega_{n}^{2}\right)-j \frac{\omega}{\tau_{a}}\right]\left[\left(\omega^{2}-\omega_{n^{\prime}}^{2}\right)+j \frac{\omega}{\tau_{a}}\right]}\right. \\
& \left.+\sum_{n=n^{\prime}} \frac{\delta \phi_{n}^{2} \phi_{n}\left(\mathbf{r}_{l}^{R}\right) \phi_{n}\left(\mathbf{r}_{l^{\prime}}^{R}\right)}{\left(\omega_{n}^{2}\right)^{2}+\frac{\omega^{2}}{\tau_{a}^{2}}}\right) \frac{\left\langle|q(\omega)|^{2}\right\rangle}{\rho_{s}^{2}},
\end{aligned}
$$

with $\delta \phi_{n}^{2}=\frac{1}{N_{S}} \sum_{k}\left|\phi_{n}\left(\mathbf{r}_{k}^{S}\right)\right|^{2}-\overline{\phi^{2}}$.

The noise correlation function being the inverse Fourier transform of the cross-spectral density matrix given by (5), it can be expressed as

$$
C_{l l^{\prime}}(t)=\left(G\left(\mathbf{r}_{l}^{R}, \mathbf{r}_{l^{\prime}}^{R}, t\right)-G\left(\mathbf{r}_{l}^{R}, \mathbf{r}_{l^{\prime}}^{R},-t\right)\right) \otimes f(t)+n_{l l^{\prime}}(t),
$$

where $\otimes$ is the convolution, $n_{l l^{\prime}}$ is the inverse Fourier transform of $N$ and

$$
f(t)=\frac{N_{S} \overline{\phi^{2}} \tau_{a}}{2 \rho_{s}} \int_{-\infty}^{t} q(\tau) \otimes q(-\tau) d \tau .
$$

When the noise is sufficiently broadband and the positions $\mathbf{r}_{l}^{R}$ and $\mathbf{r}_{l^{\prime}}^{R}$ sufficiently distant, the causal $G\left(\mathbf{r}_{l}^{R}, \mathbf{r}_{l^{\prime}}^{R}, t\right) \otimes$ $f(t)$ and anticausal $G\left(\mathbf{r}_{l}^{R}, \mathbf{r}_{l^{\prime}}^{R},-t\right) \otimes f(t)$ responses do not overlap in time. In such a case, the positive time of the cross correlation function can be interpreted as the transient response between the two positions $\mathbf{r}_{l}^{R}$ and $\mathbf{r}_{l^{\prime}}^{R}$ when at one position, a time dependent load $f(t)$ excites the plate. However, because the noise is generated by only a finite number $N_{S}$ of noise sources, the additive parasitic term $n_{l l^{\prime}}(t)$ degrades the Green's function extraction. It should be noted indeed that if the noise sources are uniformly distributed, $n_{l l^{\prime}}(t)$ is zero because $\delta \phi_{n}^{2}=0$ and $\sum_{k} \phi_{n}\left(\mathbf{r}_{k}^{S}\right) \phi_{n^{\prime}}\left(\mathbf{r}_{k}^{S}\right) \propto \delta_{n, n^{\prime}}$. In that case, we find the classical result that when the noise sources are uniformly distributed, the time derivative of the cross-correlation yields the difference between the causal and anti-causal Green's function.

\section{B. Description of the numerical simulation}

We use the finite element software Elmer ${ }^{24}$ to simulate the propagation of the flexural wave on a thin plate. In Fig. 1 the configuration of the numerical simulation is shown. We consider a rectangular aluminum plate of dimensions $0.5 \times 0.3 \mathrm{~m}^{2}$ and $3 \mathrm{~mm}$ of thickness. The reference state (without defect) is defined as the case when the material properties (Young's modulus, Poisson's ratio, the volume density, and local plate thickness) are uniform, whereas a defect is simulated by a local modification of these material properties. The sources considered in the simulation are a time-dependent normal load $q(t)$. The working frequency band of the sources is between $10 \mathrm{kHz}$ and $30 \mathrm{kHz}$ where the $\mathrm{A}_{0}$ Lamb mode dominates. We choose a simulation time step of $2 \mu \mathrm{s}$ and a spatial step of approximately $3.5 \mathrm{~mm}$ which corresponds to a tenth of the central frequency wavelength. The source $\left(S_{k}\right)$ and receiver $\left(R_{l}\right)$ positions are picked at random (see Fig. 1). Since FDTD numerical simulation is very time consuming, instead of continuous noises, the sources emit successively identical short pulses. From $N_{S}$ simulation runs, we obtain the $N_{S} \times N_{R}$ transient normal responses

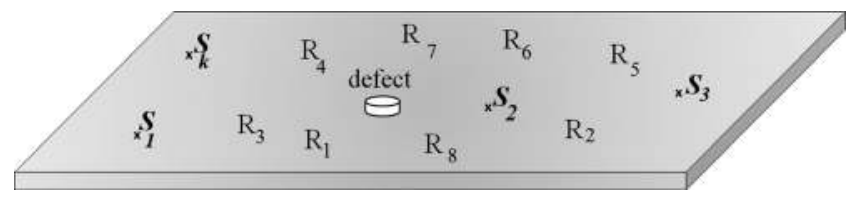

FIG. 1. Description of the simulated set-up. 
$h\left(\mathbf{r}_{k}^{S}, \mathbf{r}_{l}^{R}, t\right)$. From this set of signals, we directly estimate the cross correlations

$$
C_{l l^{\prime}}(t)=\sum_{k} h\left(\mathbf{r}_{k}^{S}, \mathbf{r}_{l}^{R}, t\right) \otimes h\left(\mathbf{r}_{k}^{S}, \mathbf{r}_{l^{\prime}}^{R},-t\right) .
$$

Since $h\left(\mathbf{r}_{k}^{S}, \mathbf{r}_{l}^{R}, t\right)=G\left(\mathbf{r}_{k}^{S}, \mathbf{r}_{l}^{R}, t\right) \otimes q(t)$, this expression is equivalent to Eq. (3) assuming that the noise autocorrelation is equal to $q(t) \otimes q(-t)$. Hence, we simulate the case of perfect uncorrelated noise sources that emit the same power spectrum.

To compare the cross correlation function to the Green's function we also estimate the transient responses $h\left(\mathbf{r}_{l}^{R}, \mathbf{r}_{l^{\prime}}^{R}, t\right)$ between the receiver positions. From this set of responses, we construct the transient response matrix $\mathbf{h}(t)$.

In Fig. 2(a), the causal part (positive times) of the autocorrelation $C_{11}(t)$ for the case of 20 sources randomly distributed is compared to the transient backscattered response $h\left(\mathbf{r}_{1}^{R}, \mathbf{r}_{1}^{R}, t\right)$ at position $\mathbf{r}_{1}^{R}$. This figure shows that the general features of the propagation from $\mathbf{r}_{1}^{R}$ to itself are retrieved by the correlation process, with the different wavepackets corresponding, respectively, to the direct path and the random-like part associated to the multiple reflections from the plate boundaries. However, a time shift is observed between both signals. This is due to the difference between $f(t)$ of Eq. (6) and $q(t)$. Contrary to $q(t)$, signal $f(t)$ is an antisymmetric signal centered at $t=0$.

To quantitatively estimate the difference between the cross correlation and the transient response, we plot $h\left(\mathbf{r}_{1}^{R}, \mathbf{r}_{1}^{R}, t\right) \otimes \int g(-t) d t$ in Fig. 2(b), where $g(t)=\frac{N_{S} \overline{\phi^{2}} \tau_{a}}{2 \rho_{s}} q(t)$.
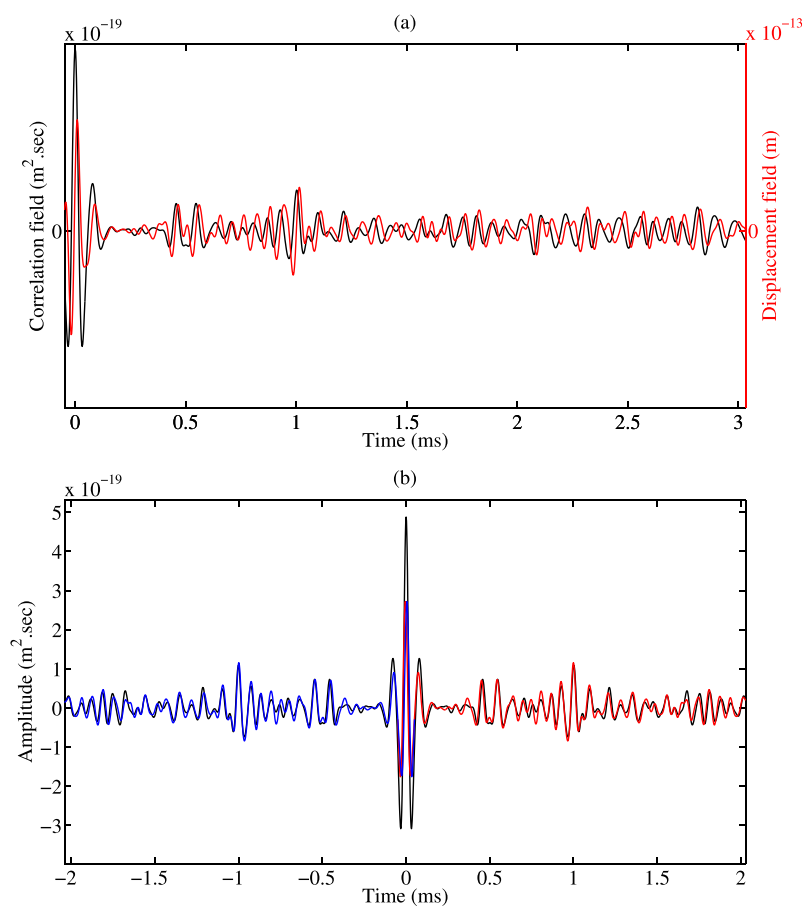

FIG. 2. (a) Comparison between the averaged correlations $C_{11}(t)$ over 20 random source positions (black curve) and the transient back-scattered response $h\left(\mathbf{r}_{1}^{R}, \mathbf{r}_{1}^{R}, t\right)$ (red curve), (b) Comparison between the averaged correlations $C_{11}(t)$ over 20 random source positions (black curve), the corrected causal response $h\left(\mathbf{r}_{1}^{R}, \mathbf{r}_{1}^{R}, t\right) \otimes \int g(-t) d t$ (red curve) and the corrected anticausal response $h\left(\mathbf{r}_{1}^{R}, \mathbf{r}_{1}^{R},-t\right) \otimes \int g(t) d t$ (blue curve).
For time $|t|>T_{0}$, where $T_{0}$ is the inverse of the working bandwidth, the agreement with the autocorrelation is excellent in both phase and amplitude either for the anticausal response or the causal one. Indeed, in such a case, the causal and anticausal parts of the autocorrelation do not overlap. On the contrary, when $|t|<T_{0}$, the correlation results from the interference between them. Thus at $t=0$, the correlation is dominated by the imaginary part of $G\left(\mathbf{r}_{1}^{R}, \mathbf{r}_{1}^{R}, \omega\right)$ estimated at the central working frequency. Contrary to the membrane Green's function (that is, solution of the Helmoltz equation), the Green's function of flexural wave is not singular at the origin. ${ }^{25}$ Moreover, the real part of $G\left(\mathbf{r}_{1}^{R}, \mathbf{r}_{1}^{R}, \omega\right)$ is null. That is why the amplitude of correlation field is twice higher than the amplitude of the causal (or anticausal) response at the origin. This numerical result is a confirmation of the validity of Eq. (6). The slight difference is due to the incoherent field term $n_{l l^{\prime}}(t)$ that is due to the non-perfect convergence of the correlations towards the Green's functions.

In strong reverberating media, a weak defect will induce only a very tiny modification of the acoustic response. Assuming an acquisition system with sufficiently high dynamic and sensitivity, the subtraction of the acoustic responses with and without defect will reveal the difference. This differential approach is perfectly adapted to SHM where one monitors the apparition of defects. We therefore consider the differential matrices $\Delta \mathbf{h}$ and $\Delta \mathbf{C}$, whose elements are defined as

$$
\begin{aligned}
\Delta h_{i j}(t) & =h^{\operatorname{def}}\left(\mathbf{r}_{i}^{R}, \mathbf{r}_{j}^{R}, t\right)-h^{\operatorname{ref}}\left(\mathbf{r}_{i}^{R}, \mathbf{r}_{j}^{R}, t\right), \\
\Delta C_{i j}(t) & =C^{\operatorname{def}}\left(\mathbf{r}_{i}^{R}, \mathbf{r}_{j}^{R}, t\right)-C^{\operatorname{ref}}\left(\mathbf{r}_{i}^{R}, \mathbf{r}_{j}^{R}, t\right),
\end{aligned}
$$

where the upper scripts ${ }^{\text {def }}$ and ${ }^{\text {ref }}$ denote the cases with and without defect, respectively.

Here, the defect is a blind hole. It is a circular region of diameter $0.5 \mathrm{~cm}$ and $3 \mathrm{~mm}$ of thickness, introduced in the finite element meshing (see Fig. 1). In Fig. 3, an example of comparison between $\Delta h_{27}(t) \otimes \int g(-t) d t$ and $\Delta C_{27}(t)$ is shown. We clearly observe on $\Delta h_{27}(t) \otimes \int g(-t) d t$ the echo of the first reflection on the defect that arrives at time $350 \mu \mathrm{s}$. However, the term $n_{l l^{\prime}}(t)$ in Eq. (6) appears to overcome the defect echo on the noise correlation function. Nevertheless, it will be shown in Sec. III that thanks to the incoherent structure of $n_{l l^{\prime}}(t)$, we can mitigate its contribution by performing spatial matched-filtering and localize the defect.

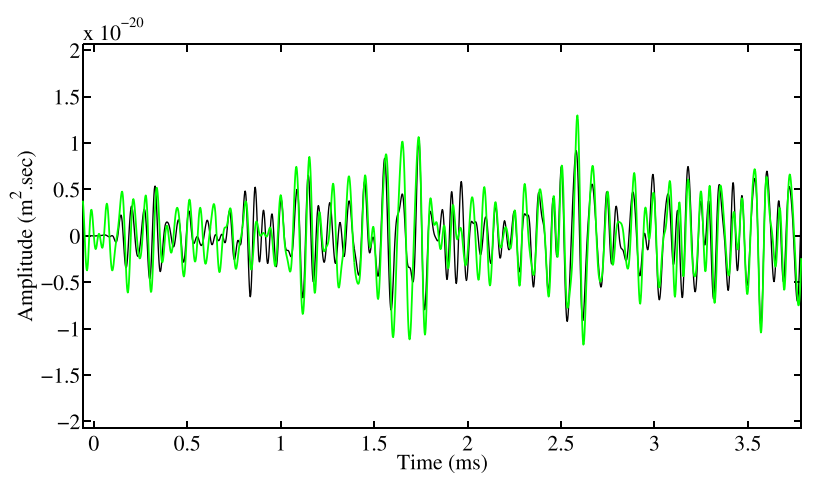

FIG. 3. Comparison between $\Delta h_{27}(t) \otimes \int g(-t) d t$ (black curve) and $\Delta C_{27}(t)$ (green curve). 


\section{LOCALIZATION OF THE DEFECT}

In this section, we apply an algorithm that is intended both to minimize the non-coherent parts in the correlation functions, while finding the defect position.

\section{A. Description of the localization algorithm}

The principle of the algorithm used in the following is similar to what is described, for example, by Michaels and Michaels, ${ }^{26}$ except here Lamb wave dispersion is taken into account. The principle is to take the element of indexes $(i, j)$ of either the $\Delta \mathbf{h}$ (active case) or $\Delta \mathbf{C}$ (passive case) matrices and back-propagate it according to the current pixel position $(x, y)$. The back-propagation function $(b p f)$ of $\Delta \mathbf{h}$ is estimated from

$b p f_{(x, y)}(\omega)=\sum_{i=1, j=1(i \neq j)}^{N_{R}, N_{R}} \Delta h_{i j}(\omega) \exp \left[j\left[d_{i}(x, y)+d_{j}(x, y)\right] k(\omega)\right]$,

where $d_{i}(x, y)$ is the distance between the $i$ th receiver and the pixel at position $(x, y)$ (Fig. 4). The wavenumber $k$ obeys to the dispersion relation of $\mathrm{A}_{0}$ mode. Matrix element $\Delta h_{i j}(\omega)$ is the Fourier transform of $\Delta h_{i j}(t)$. Only the time interval that includes the direct propagation between the defect and the receivers is kept. In practice, due to the electronic setup, the response of the transducers toward themselves cannot be measured. For this reason, the diagonal elements $\Delta h_{i i}$ are not considered to compute $b p f$.

After returning to the time domain through an inverse Fourier transform, the pixel intensity at position $(x, y)$ is then obtained by integrating the back-propagation function over time $T_{0}$.

$$
I(x, y)=\int_{-T_{0 / 2}}^{T_{0} / 2}\left|b p f_{(x, y)}(t)\right|^{2} d t
$$

where $T_{0}$ is typically the inverse of the bandwidth. For pixels located on the defect, this process will be equivalent to a numerical backpropagation of the signals to zero time (instant of the emission) followed by a coherent summation of the backpropagated signals. This will lead to a constructive sum and a maximum of the pixel intensity. As for pixels located elsewhere, the obtained intensity will be made up of a summation of non-coherent contributions corresponding both to the reverberations at the plate boundaries and, in the passive imaging case, the correlation residue $n_{l l^{\prime}}(t)$. Exactly the same

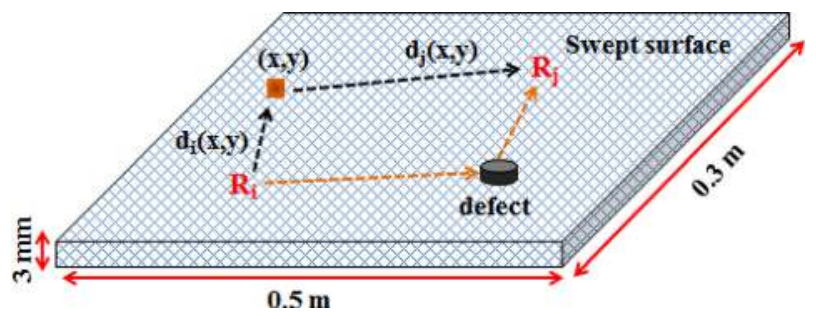

FIG. 4. Illustration of the localization algorithm principle.

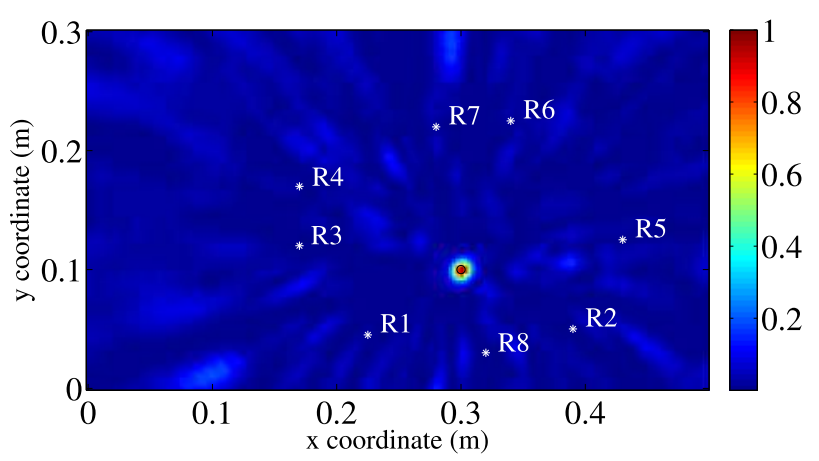

FIG. 5. Defect localization image obtained from $\Delta \mathbf{h}(t)$

procedure can be performed by replacing $\Delta \mathbf{h}(t)$ by the causal part of $\Delta \mathbf{C}(t)$. But, contrary to the active case, the diagonal elements of $\Delta \mathbf{C}(t)$ (the autocorrelations) are taken into account to work out the $b p f$.

\section{B. Localization results}

The localization algorithm is applied to the same numerical test-case as described above. The defect coordinates are $(0.3 \mathrm{~m}, 0.1 \mathrm{~m})$, with the origin taken at the left bottom corner of the plate, and its location is indicated by a small black circle on Figs. 5-7. The locations of the receivers $\mathrm{R}_{i}$ are indicated by white crosses.

First, the "active" signal matrix $\Delta \mathbf{h}(t)$ is used as an input of the algorithm. The resulting image (Fig. 5) shows a clear spot on the defect location. Since the plate is of finite dimensions, additional noise associated to the reverberated echoes is inevitably observed. Indeed, since the knowledge of the plate properties and geometry would possibly be imperfect in a practical application, the measured field is backpropagated as though it were acquired in an unbounded plate. This will limit the impact of slight discrepancies in the plate properties (in particular the wavenumber $k$ ), because the involved distances will remain moderate. In such a case, however, the few reflections that would be included in the beginning of $\Delta h_{i j}(t)$ will produce secondary lobes (i.e., spurious spots on the image). Still, the contrast of the image is very good.

Second, the algorithm is applied to the correlation matrix $\Delta \mathbf{C}(t)$ averaged over 20 random source positions (Fig. 6). The image quality is naturally lower than in the

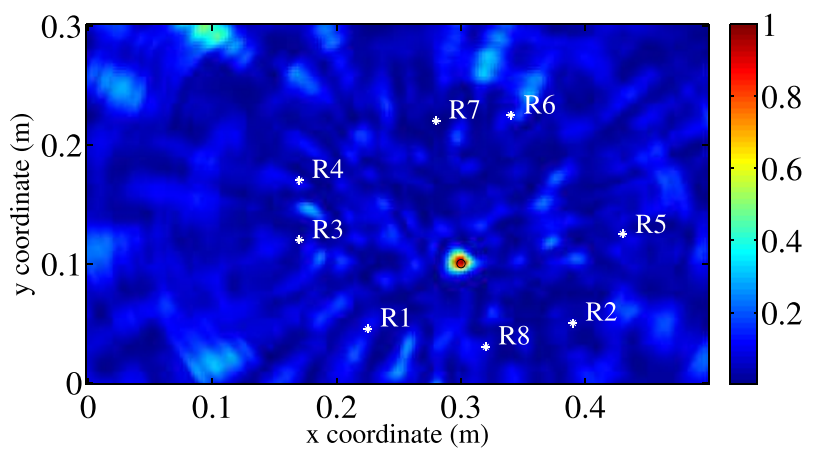

FIG. 6. Defect localization image obtained from $\Delta \mathbf{C}(t)$ (20 random sources). 


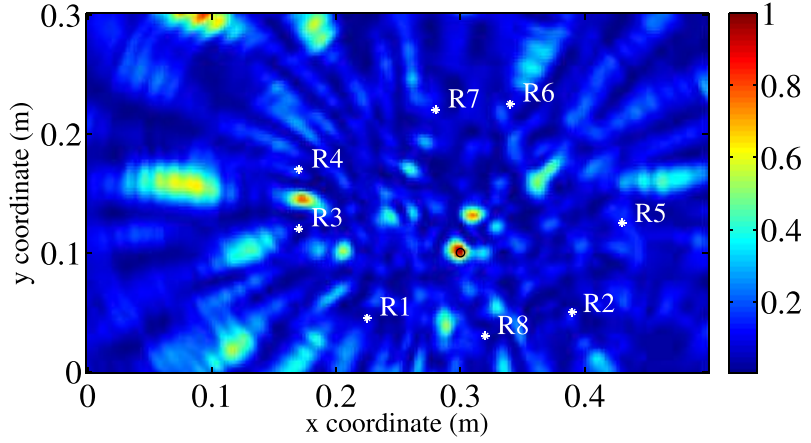

FIG. 7. Defect localization image obtained from $\Delta \mathbf{C}(t)$ (3 random sources).

active case. However, the canceling of the non-coherent parts outside the defect is sufficiently efficient to make the defect clearly visible.

Finally, in order to test a more challenging case, Fig. 7 shows the image obtained with a correlation matrix constructed from only 3 random sources. Though the defect is still visible, additional secondary lobes of equivalent amplitudes are present.

In Sec. III C, it will be shown how the DORT method can be applied to filter the correlation matrices in order to reduce the level of secondary lobes.

\section{Filtering using the DORT method}

One application of the DORT method is to reduce the effect of noise when one wants to detect/localize one or a few targets. Here by noise, we mean every process that generates "incoherent" fields such as electronic noise, clutter scattering, etc. For instance, in nondestructive testing, DORT method drastically decreases the contribution due to the scattering on grain in titanium that scrambles the ultrasonic wave reflection off the defect. ${ }^{27}$ In underwater acoustics, DORT method cancels the reverberation due to scattering on rough sea floor. ${ }^{28}$

Here, there are two main sources of "noise" that are added to the imaginary part of the Green's function. First when the correlation is not sufficiently averaged over time, i.e., the noise is not integrated over a sufficiently long time windows, the sources of noise are seen as correlated and it adds to Eq. (3), a double sum over each pair of random sources. Second, even when the random sources are uncorrelated, a noise term $n_{l l^{\prime}}$ in Eq. (6) is present because there is only a finite number of random sources. To take benefit of DORT method, a singular value decomposition (SVD) is applied to the Fourier transforms of the (time-domain) propagation and correlation matrices $\Delta \mathbf{H}(\omega)$ and $\Delta \mathbf{C}(\omega)$, respectively. For each frequency $\omega$, SVD can be written as

$$
\Delta \mathbf{H}(\omega)=\sum_{i=1}^{N_{R}} \mathbf{U}_{\Delta H}^{i}(\omega) S_{\Delta H}^{i}(\omega) \tilde{\mathbf{V}}_{\Delta H}^{i}(\omega),
$$

where ${ }^{\sim}$ stands for the Hermitian conjugation, $\mathbf{U}_{\Delta H}^{i}$ and $\mathbf{V}_{\Delta H}^{i}$ are the $i$ th singular vector associated to the $i$ th singular value $S_{\Delta H}^{i}$. The same decomposition can be operated on $\Delta \mathbf{C}(\omega)$,

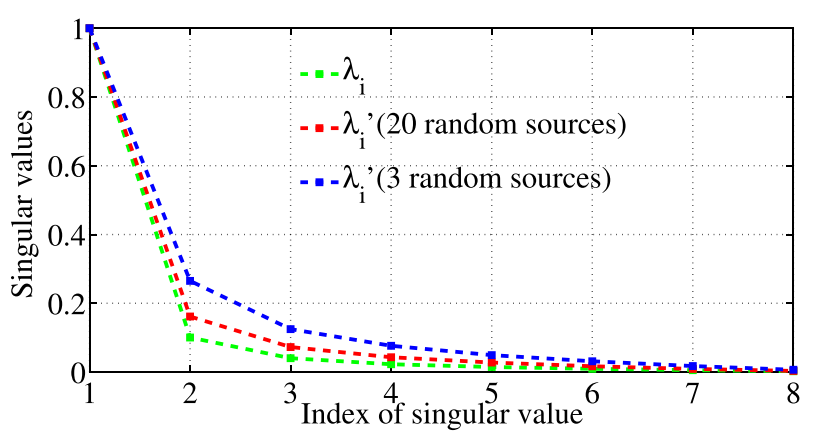

FIG. 8. Normalized and averaged over frequencies singular values of, respectively: $\Delta \mathbf{H}(\omega)$ (green curve), $\Delta \mathbf{C}(\omega)$ with 20 random sources (red curve) and $\Delta \mathbf{C}(\omega)$ with 3 random sources (blue curve).

$$
\Delta \mathbf{C}(\omega)=\sum_{i=1}^{N_{R}} \mathbf{U}_{\Delta C}^{i}(\omega) S_{\Delta C}^{i}(\omega) \tilde{\mathbf{V}}_{\Delta C}^{i}(\omega)
$$

Figure 8 represents the singular value distribution averaged over the whole frequency range and normalized according to the first one. The green curve shows that the first singular value $\lambda_{1}$ is largely dominant and therefore $\Delta \mathbf{H}(\omega)$ is close to a rank-one matrix. This is a typical result obtained with the DORT method when a single scatterer is considered, which is the case here whenever the defect-free response is subtracted from the response with the defect. This means concretely that the information about the scatterer is essentially contained in the first singular vector.

To confirm this result, we back-propagate the first eigen-vector, i.e.,

$$
\begin{aligned}
\operatorname{sbp}_{(x, y)}(\omega)= & S_{\Delta H}^{1}\left(\sum_{i}\left[U_{\Delta \mathbf{H}}^{1}\right]_{i} e^{j d_{i}(x, y) k}\right) \\
& \times\left(\sum_{i}\left[\tilde{V}_{\Delta \mathbf{H}}^{1}\right]_{i} e^{j d_{i}(x, y) k}\right) .
\end{aligned}
$$

Actually, we have constructed in this way a filtered version of $\Delta \mathbf{H}(\omega)$, in which the contributions of all singular vectors except the first one are canceled. As expected, the application of the localization algorithm to this filtered matrix provides a very similar result (Fig. 9) to the obtained image in Fig. 5 because the rank of $\Delta \mathbf{H}(\omega)$ was almost equal to 1 .

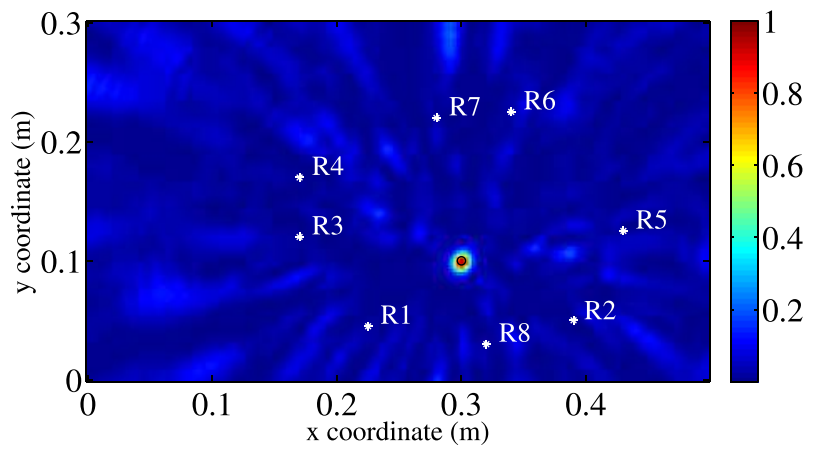

FIG. 9. Defect localization image obtained from the filtered version of $\Delta \mathbf{H}(\omega)$. 


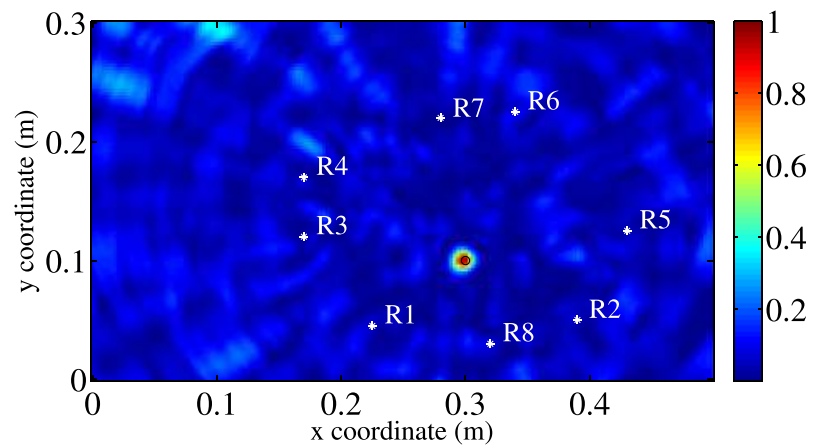

FIG. 10. Defect localization image obtained from the filtered version of $\Delta \mathbf{C}(\omega)$ (20 random sources).

As for $\Delta \mathbf{C}(\omega)$, though the first singular value $\lambda_{1}^{\prime}$ is here again dominant over the next ones (see red and blue curves in Fig. 8), the ratio between it and the next ones is significantly lower than in the case of $\Delta \mathbf{H}(\omega)$. Especially for 3 random sources, where it appears almost three times lower. This behavior is a direct consequence of the imperfect reconstruction of the Green's functions by the correlation process. Indeed, as already shown in Sec. II, the correlation matrix $\Delta \mathbf{C}(\omega)$ can be seen as a replica of $\Delta \mathbf{H}(\omega)$, scrambled by a non-coherent residual part. Then the lower the number of random sources, the less accurate the Green's functions reconstruction, the more $\Delta \mathbf{C}(\omega)$ differ from a rank-one matrix. In these conditions, only keeping the most significant eigen-value from $\Delta \mathbf{C}(\omega)$ to construct the $b p f$ should remove a large part of the incoherent noise and improve the target localization. Indeed, one can show that ${ }^{29}$ assuming the noise level is a fraction $\epsilon$ of the signal one $(\epsilon<1)$, the amplitude of the side lobes compared to the main one when the bpf is classically computed [Eq. (8)] is proportional to $\epsilon$ whereas it drops to $\epsilon^{2}$ when only the first eigenvector is used [Eq. (12)].

Figures 10 and 11 show the images obtained from filtered $s b p$ function for 20 and 3 random source positions, respectively. In the case of 20 random sources, the improvement of the image quality (compared to the image of Fig. 6) is marginal because the reconstruction was already fairly good.

The case of 3 random sources is more illustrative. A comparison between Figs. 11 and 7 shows a clear reduction of the spurious spots and therefore a better localization of the defect. The imperfections that remain on the image are due

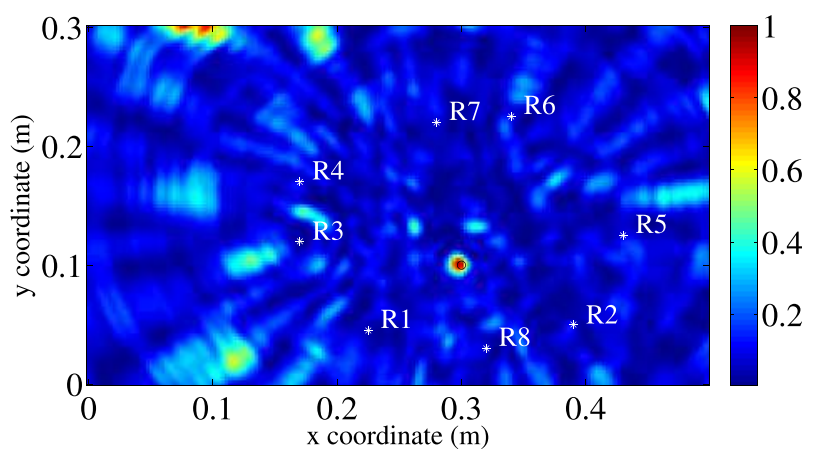

FIG. 11. Defect localization image obtained from the filtered version of $\Delta \mathbf{C}(\omega)$ (3 random sources). to the residual noise that still affected a little the first singular vector of the correlation matrix.

\section{CONCLUSION}

In this paper, the theoretical relationship between the correlation of signals received on a set of sensors and the Green's function in a reverberant plate has been established. This has allowed to introduce a simple means to quantitatively compare the correlations to the emission-reception responses. It is shown, in particular, that even in conditions where the correlations converge fairly well towards the active responses, it might not be so in a differential mode (differences between the case with defect and the healthy case). Despite this non-perfect convergence of the differential correlations, application of a backpropagation and coherent summation algorithm has allowed the detection and localization of a defect. Indeed, the algorithm has been demonstrated to be efficient not only in localizing the defect but also in reducing sufficiently the anomalies in the Green's functions reconstruction. Finally, we have shown the efficiency of the DORT method for filtering the differential correlation matrices with as few as three random sources.

\section{ACKNOWLEDGMENTS}

This work has been supported by the French National Research Agency (ANR): No. ANR2011 BS0903901, PASNI Project.

${ }^{1}$ W. Luo, J. L. Rose, and H. Gao, "A peak frequency shift method for guided wave thickness measurement and its realization by different transducer techniques," in 16th WCNDT 2004-World Conference on NDT, 30 August-September 3, 2004-Montreal, Canada, 2004.

${ }^{2}$ A. Pilarski and J. L. Rose, "Lamb wave mode selection concepts for interfacial weakness analysis," Nondestr. Eval. 11, 237-249 (1992).

${ }^{3}$ J.-B. Ihn and F.-K. Chang, "Pitch-catch active sensing methods in structural health monitoring for aircraft structures," Struct. Health Monit. 7, 5-19 (2008).

${ }^{4}$ P. Gueguen, M. Langlais, P. Foray, C. Rousseau, and J. Maury, “A natural seismic isolating system: The buried mangrove effects," Bull. Seismol. Soc. Am. 101(3), 1073-1080 (2011).

${ }^{5} \mathrm{M}$. Campillo and A. Paul, "Long-range correlations in the diffuse seismic coda," Science 299, 547-549 (2003).

${ }^{6}$ N. M. Shapiro and M. Campillo, "Emergence of broadband Rayleigh waves from correlations of the ambient seismic noise," Geophys. Res. Lett. 31, L07614, doi:10.1029/2004GL019491 (2004).

${ }^{7}$ T. Gallot, "Imagerie acoustique en milieux reverberants," Ph.D. dissertation (Universite de Grenoble, 2010).

${ }^{8}$ K. G. Sabra, E. S. Winkel, D. A. Bourgoyne, B. R. Elbing, S. L. Ceccio, M. Perlin, and D. R. Dowling, "Using cross correlations of turbulent flowinduced ambient vibrations to estimate the structural impulse response. Application to structural health monitoring," J. Acoust. Soc. Am. 121, 1987 (2007).

${ }^{9}$ P. Roux, W. A. Kuperman, and The NPAL Group, "Extracting coherent wavefronts from acoustic ambient noise in the ocean," J. Acoust. Soc. Am. 116, 1995-2003 (2004).

${ }^{10}$ F. Brenguier, D. Clarke, Y. Aoki, N. M. Shapiro, M. Campillo, and V. Ferrazzini, "Monitoring volcanoes using seismic noise correlations," C. R. Geosci. 343(8), 633-638 (2011).

${ }^{11}$ R. L. Weaver and O. I. Lobkis, "Ultrasonics without a source: Thermal fluctuation correlations at mhz frequencies," Phys. Rev. Lett. 87(13), 134301 (2001).

${ }^{12}$ R. L. Weaver and O. I. Lobkis, "Elastic wave thermal fluctuations, ultrasonic waveforms by correlation of thermal phonons," J. Acoust. Soc. Am. 113, 2611 (2003). 
${ }^{13}$ A. Derode, E. Larose, M. Tanter, J. de Rosny, A. Tourin, M. Campillo, and M. Fink, "Recovering the green's function from field-field correlations in an open scattering medium," J. Acoust. Soc. Am. 113, 2973 (2003).

${ }^{14}$ S. M. Efferies, M. A. Pomerantz, T. L. Duvall, and J. W. Harvey, Jr., "Helioseismology from the south pole: Closer connections with geoseismology," Antarct. J. 28(5), 328-329 (1993).

${ }^{15}$ E. Larose, O. I. Lobkis, and R. L. Weaver, "Passive correlation imaging of a buried scatterer," J. Acoust. Soc. Am. 119, 3549-3552 (2006).

${ }^{16}$ N. A. Leyla, E. Moulin, and J. Assaad, "Influence of a localized defect on acoustic field correlation in a reverberant medium," J. Appl. Phys. 110(8), 084906 (2011).

${ }^{17}$ E. Moulin, N. A. Leyla, J. Assaad, and S. Grondel, "Applicability of acoustic noise correlation for structural health monitoring in nondiffuse field conditions," Appl. Phys. Lett. 95(9) 094104 (2009).

${ }^{18}$ C. Prada and J. L. Thomas, "Experimental sub-wavelength localization of scatterers by decomposition of the time reversal operator interpreted as a covariance matrix," J. Acoust. Soc. Am. 114, 235-243 (2003).

${ }^{19}$ J.-G. Minonzio, F.-D. Philippe, C. Prada, and M. Fink, "Characterization of elastic cylinder and sphere with the time-reversal operator: Application to the sub-resolution limit," Inverse Probl. 24, 025014 (2008).

${ }^{20}$ C. Prada, S. Manneville, D. Spoliansky, and M. Fink, "Decomposition of the time reversal operator: Detection and selective focusing on two scatterers," J. Acoust. Soc. Am. 99, 2067 (1996).
${ }^{21}$ O. I. Lobkis and R. L. Weaver, "On the emergence of the green's function in the correlations of a diffuse field," J. Acoust. Soc. Am. 110, 3011 (2001).

${ }^{22}$ A. E. H. Love, "The small free vibrations and deformation of a thin elastic shell," Philos. Trans. R. Soc., A 179, 491-546 (1888).

${ }^{23}$ G. Barton, Elements of Green's Functions and Propagation: Potentials, Diffusion, and Waves (Oxford Science Publications, Clarendon Press, 1989).

${ }^{24}$ Elmer is an open source multiphysical simulation software mainly developed by CSC - IT Center for Science (CSC). It includes Finite Element solution of plate equations. See http://www.csc.fi/english/pages/elmer.

${ }^{25}$ For unbounded plates, the monochromatic Green's function is equal to $\frac{1}{D 8 k^{2}}\left[Y_{0}(k R)+\frac{2}{\pi} K_{0}(k R)+j J_{0}(k R)\right]$ where $k^{4}=\frac{\rho_{S} \omega^{2}}{D}$ and $R$ is the distance between the two position entries of the Green's function.

${ }^{26} \mathrm{~J}$. E. Michaels and T. E. Michaels, "Guided wave signal processing and image fusion for in situ damage localization in plates," Wave Motion 44, 482-492 (2007).

${ }^{27}$ C. Prada, E. Kerbrat, D. Cassereau, and M. Fink, "Time reversal techniques in ultrasonic nondestructive testing of scattering media," Inverse Probl. 18(6), 1761 (2002).

${ }^{28}$ C. Prada, J. de Rosny, D. Clorennec, J.-G. Minonzio, A. Aubry, M. Fink, L. Berniere, P. Billand, S. Hibral, and T. Folegot, "Experimental detection and focusing in shallow water by decomposition of the time reversal operator," J. Acoust. Soc. Am. 122, 761 (2007).

${ }^{29}$ The demonstration is out of the scope of the current paper. An article devoted to this particular point is in preparation. 